\title{
Summary of 'Demographic and human capital scenarios for the 21st century: 2018 assessment for 201 countries'
}

\author{
Nicholas Gailey and Wolfgang Lutz*
}

\begin{abstract}
In 2016, the Joint Research Centre (JRC) of the European Commission and the International Institute for Applied Systems Analysis (IIASA) agreed to form a partnership, establishing the Centre of Expertise on Population and Migration (CEPAM). The work presented here summarises the first results published by CEPAM. The results reveal clear momentum towards population ageing, and how migration has limited ability to influence the population structure of the EU, especially in the long-run. On the other hand, boosting labour force participation can nullify expected rises in the dependency ratio from population ageing. Globally, the findings show the future of population growth and socio-economic development will be determined by the expansion of education, particularly among girls in Africa. Scenarios of either rapid or stalled development illustrate a large range of possible futures for world population by 2100 .
\end{abstract}

\section{Introduction}

Any meaningful understanding of the future world hinges on informed projections of how current demographic trends could unfold. In that spirit, the Centre of Expertise on Population and Migration (CEPAM) has developed contrasting scenarios based on the most recent demographic data, analyses, and trajectories

\footnotetext{
* Nicholas Gailey (corresponding author), Wittgenstein Centre for Demography and Global Human Capital (IIASA, VID/ÖAW, WU), International Institute for Applied Systems Analysis, Schlossplatz 1, 2361 Laxenburg, Austria

Email: gailey@iiasa.ac.at

Wolfgang Lutz, Wittgenstein Centre for Demography and Global Human Capital (IIASA, VID/ÖAW, WU), Vienna, Austria
} 
for 201 countries. The scenarios cover key population and human capital measures, looking as far into the future as the century's end.

By considering not only age and sex structures, but also educational attainment (in all countries) and labour force participation (in EU member states), this CEPAM volume goes beyond the more limited ambitions of conventional population projections. The results account for key drivers of change and thus help to paint a more complete demographic picture. Ultimately, the purpose of CEPAM's contrasting scenarios is to inform policy-focused assessments of migration flows into the European Union.

This volume serves as an update to the population projections presented in World population and human capital in the 21 st century (Lutz et al. 2014), which provides a comprehensive summary of scientific arguments about assumptions for future fertility, mortality, migration, and education based on input from more than 550 population experts. The baseline population numbers used in this CEPAM volume incorporate new censuses and survey data up through 2015.

In terms of migration, three rough scenarios are defined - zero migration, constant migration rates taken from the past 60-year average, and double the constant migration rate. The three strongly differing pathways were combined with medium fertility and mortality assumptions to help focus on the sensitivity of population trends to varying migration intensities. These divergent futures also serve as the basis for an expanded set of more detailed scenarios.

To project populations under varying degrees of socio-economic development, this volume also uses three 'Shared Socioeconomic Pathways', or SSPs. The SSPs compare a world of rapid social development (high education, low mortality, and low fertility) with one of stalled social development (low education, high mortality, and high fertility), and a medium variant between the two.

Almost every country in the world is now moving towards lower fertility, increasing longevity, and higher educational levels. The resulting population ageing will remain largely unavoidable for the EU, regardless of possible changes in migration or fertility. However, developments in labour force participation rates, more productive working lives, and the ongoing automation of labour all suggest a diminishing threat from population ageing. Integrating such understanding into the policy-making process is important for envisioning judicious and realistic policy goals for the future.

In this summary, CEPAM's results are presented under the following sections: EU Member States and their Future Labour Forces, Projected EU Population Size and Age Composition, and Global Population Futures.

Lastly, for abbreviated samples of the appendix tables that cover all countries (457 pages of tables in the full volume), we include Nigeria as the most populous African country, and India as the soon-to-be largest country in both Asia and the world. 
Figure 1:

Total labour force by education level in the European Union, according to constant participation - CEPAM Medium (SSP2) scenario, 2015-2060

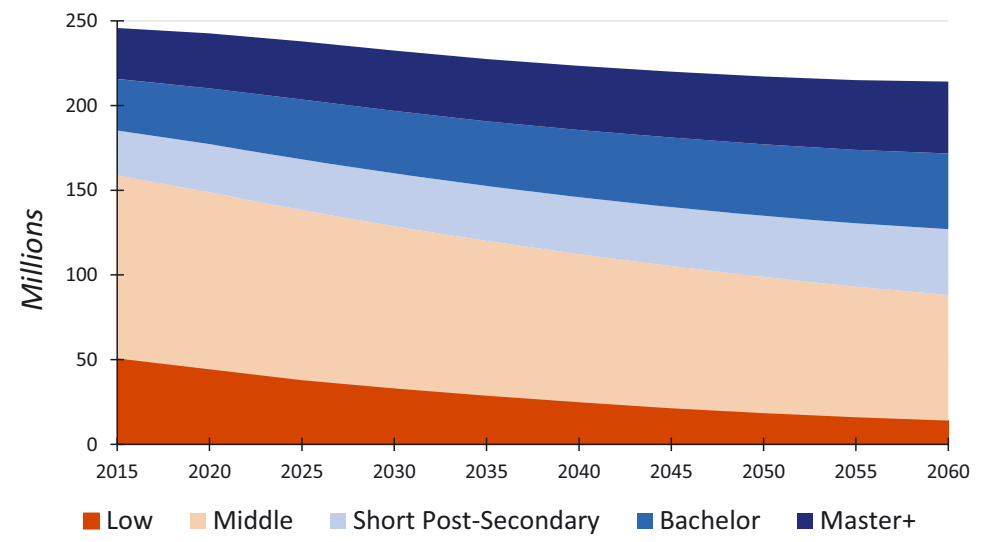

Source: Loichinger and Marois (2018).

\section{EU member states and their future labour forces}

\subsection{A smaller, higher educated labour force}

While the absolute size of the EU workforce is expected to be smaller in the coming decades, it is also likely to be more highly educated. As Figure 1 illustrates, the total size of the workforce is projected (under the CEPAM Medium scenario, SSP2 ${ }^{1}$ ) to change from 245.8 million to 214.1 million over the next four decades. The decline is solely attributable to fewer workers with educational levels of 'upper secondary' and 'lower secondary or below'. Projected decreases in the size of the labour force come from the population with education levels of low (from 50.7 to 14.0 million) and upper secondary (from 108.2 to 74.2 million).

At the same time, the numbers of workers with a master's degree or higher, a bachelor's degree, or a shorter form of post-secondary education (e.g., technical training) are all projected to increase not only in their proportions of the labour force, but also in absolute terms. Significantly, these post-secondary groups are expected to grow by almost half $(+45 \%)$ over the next 40 years.

National differences exist in the levels and rates of educational increases, but the general trend holds true across all EU countries. Irrespective of various changes to the age structure and labour force size in individual member states, it is anticipated

1 SSP2 and other Shared Socioeconomic Pathways (SSPs) are detailed in 'Global Population Futures', section 4 
that the human capital of future workers (measured by the highest level of education attainment) will be higher at every age because younger cohorts are spending more years in education than previous generations.

Looking at regional trends within the EU, we see that several eastern member states - the Czech Republic, Slovakia, Poland, Latvia, and Croatia - currently register the highest levels of post-secondary educational attainment. While CEPAM projects that all EU member states will continue moving towards higher educational attainment, Southern member states that currently have the lowest levels - i.e., Portugal, Spain, Italy, and Greece - will make the fastest gains.

In the scenario with constant participation rates, the labour force size remains stable for many western member states, due in part to the movement of workingage citizens from eastern member states. As a result of these same migration trends, the labour force size decreases in the 13 new member states (EU13). However, the labour force dependency ratios in these states would remain at intermediary levels because, on average, their populations have lower life expectancy or fewer unproductive years at older ages than their western counterparts.

Whether either population age-structures shifting towards higher ages or reductions in the working-age populations do in fact produce a smaller labour supply, depends on how age-specific labour force participation rates - particularly of women and people aged between 50 and 70 - develop in the coming decades. The extent of any economic implications also depends on labour demand, which may fundamentally shift in the coming years amid automation and other factors.

When considering future labour force size and composition, it is important not to overlook the labour force dependency ratio. This measure, together with changing productivity, in large part determines the potential economic impact of population ageing. By comparing the economically inactive population to the economically active population, the ratio captures a population's composition both in terms of demographic structure and labour force participation.

\subsection{Does Europe have a demographic need for migration?}

A recurring theme in Europe's public discussions of migration describes an unmet, ongoing demographic need that can be filled by migrants. Apprehensions revolve around the size of the future workforce and how the system might function with fewer workers. However, largely absent from such discussions are (1) considerations of existing differentials in workforce participation rates between the EU member states, specifically, important variations in female participation rates; and (2) analyses of how migration could influence the labour force dependency ratio, rather than simply the labour force size.

As can be seen in Figure 2, increasing female participation rates leads to the lowest ratio of non-workers to workers among the scenarios considered. This scenario uses observed Swedish workforce participation rates as a focal point, projecting a slow convergence of other member states towards Sweden's age- and 
Figure 2:

Six scenarios for the labour force dependency ratio of the EU, 2015-2060

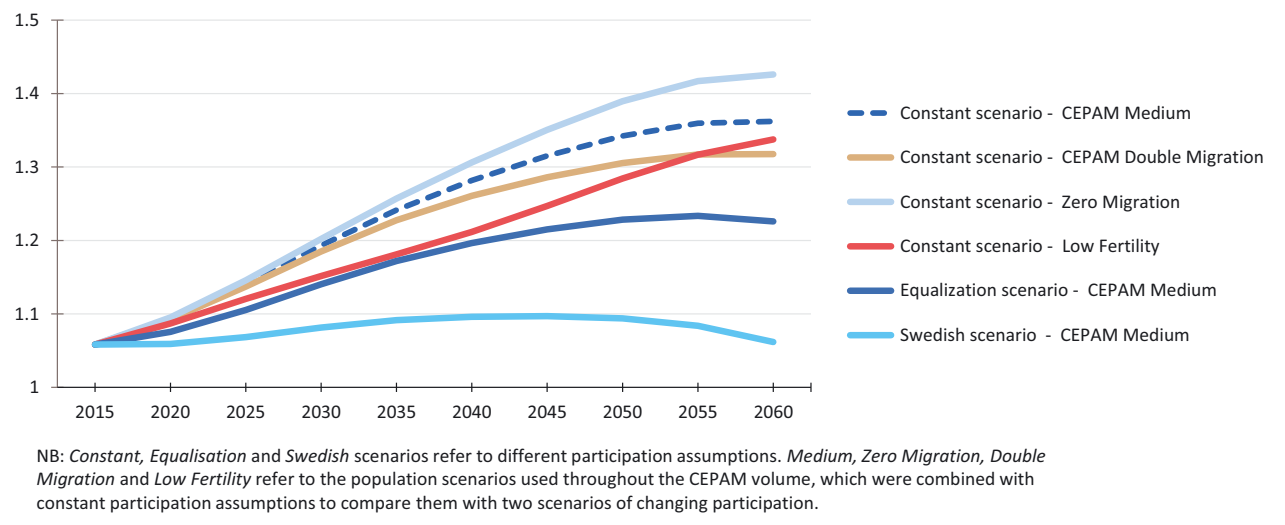

Source: Loichinger and Marois (2018)

education-specific participation rates over the course of the next 45 years. CEPAM research shows how such a gradual development would wholly counterbalance the expected rise in the dependency ratio due to population ageing.

The next-lowest dependency ratio is produced by the Equalisation scenario, in which the female labour force participation rates reach current country-specific male levels by 2060. In this situation, the expected climb in the dependency ratio would be significantly slowed, stabilising at around 1.2 dependents per worker. This amounts to about only half the increase compared to the constant scenario, arriving at nearly 1.4 dependents.

In contrast, even in the double scenario, migration leads to only a minimal decrease in the dependency ratio. CEPAM projection results show that doubling migration, despite increasing the overall population (and labour force) size, would only slightly improve the labour force dependency ratio, without altering the broader momentum towards rising levels.

Therefore, increased migration flows - particularly of migrants with low education and low labour force participation prospects - are unlikely to alleviate the economic consequences of population ageing. Although political discourse often refers to migration as a tool for easing the consequences of population ageing, demographers agree that while immigration can affect population size, it generally has little impact on the ratio of non-working to working people. Furthermore, the human capital levels of natives and migrants matter greatly for any potential improvements in productivity, and how societies will or will not cope with the consequences of automation of work processes. 


\section{Projected EU population size and age composition}

CEPAM's Medium scenario foresees the end of rapid growth in the EU-28, with the population remaining largely unchanged from the current 507.5 million to 508.3 million by the year 2060. Table 1 displays members state-specific results for population change in different scenarios.

For historical context, the population of the current EU member states (EU-28) has expanded by 128 million inhabitants since 1950. The CEPAM Zero Migration scenario finds that the rate of natural decline from low fertility brings the EU-28 population to 461 million by 2060 , or back to the size observed in the 1980s.

\subsection{Fertility growth and convergence}

Fertility in the EU-28 varies between 1.35 children per women in Portugal and two children per woman in France and Ireland (standard deviation around 0.2), with the average total fertility rate standing at 1.61 . Western members have had higher fertility levels than those in southern and eastern member states, although fertility rates appear to be slowly converging across Europe as fertility falls in the west and rebounds in the east.

According to the CEPAM Medium scenario, average period fertility in the EU will rise from the current 1.61 children per woman to 1.67 in 2030, and then to 1.71 children by 2060. In addition to higher EU-wide fertility, CEPAM Medium predicts the continuation of converging fertility behaviours across member states into the future.

\subsection{Moving west, over time - population changes from intra-EU movement}

Over time, EU internal mobility has the potential to strikingly change the demographic makeup of many member states depending on whether intensities of south- and east-to-west movements lessen, remain the same, or grow further. In some cases, net internal flows significantly impact the overall size of a sending country's population.

Particularly in eastern EU member states (Bulgaria, Croatia, the Czech Republic, Estonia, Hungary, Latvia, Lithuania, Poland, Slovakia, Slovenia, and Romania) smaller populations seem inevitable, primarily due to high net movement to western economies. Without out-migration, the populations of the eastern member states would naturally only shrink $11-25 \%$ by 2060 , similar to the natural rates of decrease in many other European countries.

If EU internal mobility continues without stabilising at lower levels, CEPAM results indicate that the most extensive population decreases reach $-38.0 \%$ for Bulgaria and $-34.3 \%$ for Romania by 2060 . In a double migration future, the 
populations of many eastern member states would fall even more dramatically, including Bulgaria $(-48.6 \%)$ and Romania $(-46.8 \%)$.

As for the proportion of people aged $65+$ in the population, higher volumes of internal mobility generally reduce age-dependency rates in western Europe and increases them in eastern Europe. If current trends continue, a particularly dynamic process will be observed in the eastern member states where lower fertility is accompanied by high volumes of movement to other EU member states, accelerating ageing.

\subsection{International migration driving population expansion in the EU}

Aside from internal movement, western member states will continue to see expanding populations if current flows of international migration either remain at constant levels or double. Such inflows would be significant enough to increase the number of people living in the EU as a whole.

Only seven countries are unlikely to naturally decreases in population size without migration (in all presented scenarios, including those of Eurostat (2015) and national projections), mostly because they have relatively high fertility: Ireland, Denmark, France, Sweden, and the UK (TFRs around 1.8-2.0 in 2015), or an age structure in which a large share of the population is of reproductive ages: Luxembourg (median age of 39.3 years) and Cyprus (35.0 years).

All CEPAM scenarios show a decrease in the populations aged 0-14 and 15-64, and an increase in the population aged $65+$. No migration leads to a reduction in the population aged $0-69$, but also to a more moderate increase in the population aged $70+$, shown in Figure 3. Whereas higher migration (CEPAM Double Migration) would reduce the magnitude of change for ages 0-69, it would also enlarge the populations aged $70+$ by 67.8 million people in the long-run, as the migrant populations inevitably grow older.

\section{Global population futures}

\subsection{The importance of education for fertility decline and longevity}

Demographic changes are to a large extent pre-determined in the near term by population age structures that result from past fertility, mortality, and migration trends. But from the longer perspective, all components of demographic change can be influenced by policy and other initiatives.

CEPAM results demonstrate that aside from policies intending to influence fertility and mortality directly, efforts to enhance education can have important indirect consequences that lead to increases in longevity and reductions in high fertility as well. The effects of socio-economic development become clear when looking at Figure 4. The two extremes in possible global development pathways 
Table 1:

Population change (in percent) in EU-28 countries from 2015-2060, by scenario

\begin{tabular}{|c|c|c|c|c|c|c|c|}
\hline \multirow[b]{2}{*}{ Country } & \multicolumn{3}{|c|}{ CEPAM } & \multirow[b]{2}{*}{ SSP1 } & \multirow[b]{2}{*}{ SSP3 } & \multirow[b]{2}{*}{ Eurostat } & \multirow[b]{2}{*}{ National } \\
\hline & $\begin{array}{c}\text { Zero } \\
\text { migration }\end{array}$ & Medium & $\begin{array}{c}\text { Double } \\
\text { migration }\end{array}$ & & & & \\
\hline Austria & -13.1 & 8.5 & 27.3 & 3.3 & -6.6 & 19.3 & 13.9 \\
\hline Belgium & -1.8 & 13.7 & 28.2 & 6.5 & 9.7 & 21.2 & 16.2 \\
\hline Bulgaria & -25.5 & -37.9 & -48.6 & -37.0 & -22.2 & -27.4 & -25.2 \\
\hline Croatia & -18.7 & -19.7 & -20.3 & -20.2 & -14.2 & -16.4 & $-16.4^{*}$ \\
\hline Cyprus & 4.2 & 23.7 & 40.9 & 21.6 & 13.8 & 19.5 & $19.5^{*}$ \\
\hline Czech Republic & -13.7 & -8.7 & -4.2 & -12.6 & -8.4 & -2.2 & -10.8 \\
\hline Denmark & 1.8 & 13.9 & 25.4 & 5.7 & 15.2 & 19.4 & 15.0 \\
\hline Estonia & -14.5 & -7.1 & -1.3 & -12.7 & -6.0 & -7.0 & $-9.2(a)$ \\
\hline Finland & 0.2 & 3.9 & 7.2 & -3.2 & 12.8 & 3.3 & 8.9 \\
\hline France & 6.1 & 18.3 & 29.1 & 9.6 & 18.8 & 13.7 & 13.2 \\
\hline Germany & -19.1 & -2.6 & 12.3 & -6.7 & -12.7 & -0.5 & -6.9 \\
\hline Greece & -15.7 & -3.5 & 7.2 & -7.2 & -10.0 & -23.6 & $0.0(b)$ \\
\hline Hungary & -22.7 & -17.9 & -13.6 & -21.0 & -18.6 & -7.5 & -19.3 \\
\hline Ireland & 16.5 & 20.8 & 24.0 & 12.1 & 27.7 & 27.4 & $26.0(\mathrm{e})$ \\
\hline Italy & -18.6 & -10.4 & -2.8 & -13.1 & -13.5 & -6.3 & -9.5 \\
\hline Latvia & -23.3 & -19.7 & -16.7 & -20.9 & -18.8 & -28.2 & $-3.4(\mathrm{c})$ \\
\hline Lithuania & -19.0 & -27.8 & -34.7 & -28.3 & -12.7 & -37.1 & $-37.1^{*}$ \\
\hline Luxembourg & 3.1 & 53.0 & 96.1 & 42.4 & 17.3 & 76.4 & 101.2 \\
\hline Malta & -10.4 & -8.9 & -7.8 & -9.5 & -5.6 & 20.9 & $20.9 *$ \\
\hline Netherlands & -2.2 & 8.6 & 18.4 & 2.2 & 8.4 & 14.3 & 5.9 \\
\hline Poland & -12.9 & -16.8 & -20.4 & -20.3 & -7.1 & -13.6 & $-11.6(d)$ \\
\hline Portugal & -17.8 & -20.3 & -22.4 & -23.2 & -13.9 & -17.6 & -17.2 \\
\hline Romania & -21.3 & -35.1 & -46.8 & -34.1 & -17.9 & -21.0 & -34.2 \\
\hline Slovakia & -10.7 & -13.6 & -16.3 & -17.2 & -5.8 & -5.7 & -1.9 \\
\hline Slovenia & -13.9 & 1.2 & 14.6 & -3.7 & -6.8 & -3.0 & -3.0 \\
\hline Spain & -13.4 & 2.6 & 17.8 & -0.7 & -7.7 & 6.7 & -8.7 \\
\hline Sweden & 7.4 & 27.0 & 45.2 & 17.6 & 20.0 & 36.3 & 30.2 \\
\hline United Kingdom & 6.1 & 17.4 & 27.2 & 8.7 & 17.4 & 22.3 & 22.9 \\
\hline EU28 & -9.1 & 0.2 & 8.6 & -4.8 & -1.3 & 3.2 & -0.9 \\
\hline
\end{tabular}

Note: $(*)$ national authorities do not prepare own projections and use Eurostat numbers, (a) projection period 2015-2040, (b) 2015-2050, (c) 2015-2030, (d) 2015-2050, (e) 2015-2045.

SSP1 = Rapid Social Development and SSP3 = Stalled Social Development.

Source: Adapted from Stonawski (2018), Eurostat, and national statistical agencies.

lead to wildly different worlds, over 5.5 billion people apart. By the end of the century, these divergent trajectories reach 7.23 billion (Rapid development/SSP1) and 13.6 billion (Stalled development/SSP3). 
Figure 3:

Change in the EU-28 population size (by age-groups) under zero, medium, and double migration conditions. * Uses in the SSP2 medium development scenario

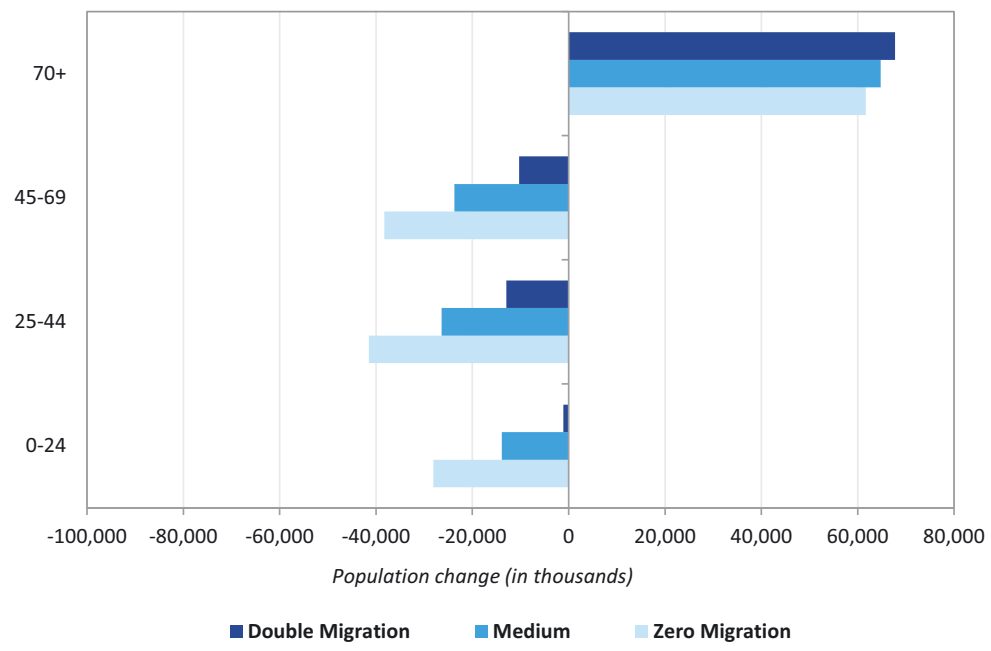

Source: Adapted from Stonawski (2018).

\footnotetext{
Shared Socioeconomic Pathways (SSPs)

SSP1 (Rapid Social Development): A sustainable and human wellbeing-focused path with an accelerated demographic transition and relatively low world population. High education, low mortality, and low fertility.

CEPAM Medium (SSP2): Middle-of-the-road scenario in which trends continue and development of low-income countries is uneven. Medium fertility, mortality, and education. For CEPAM migration projections these medium assumptions are combined with zero, medium, and double migration variants.

SSP3 (Stalled Social Development): A world separated into regions broadly characterised by rapidly growing populations. Low education, high mortality, and high fertility.
}

\subsection{Global population ageing}

Population ageing is not confined to East Asia, Europe, and North America. Under all scenarios the world population will get significantly older as a consequence of lowering fertility rates and rising life expectancy. In the CEPAM Medium (SSP2) scenario, the proportion of the population aged $65+$ increases from the current $8.3 \%$ 
Figure 4:

Comparison of different scenarios for world population growth over the 21st century

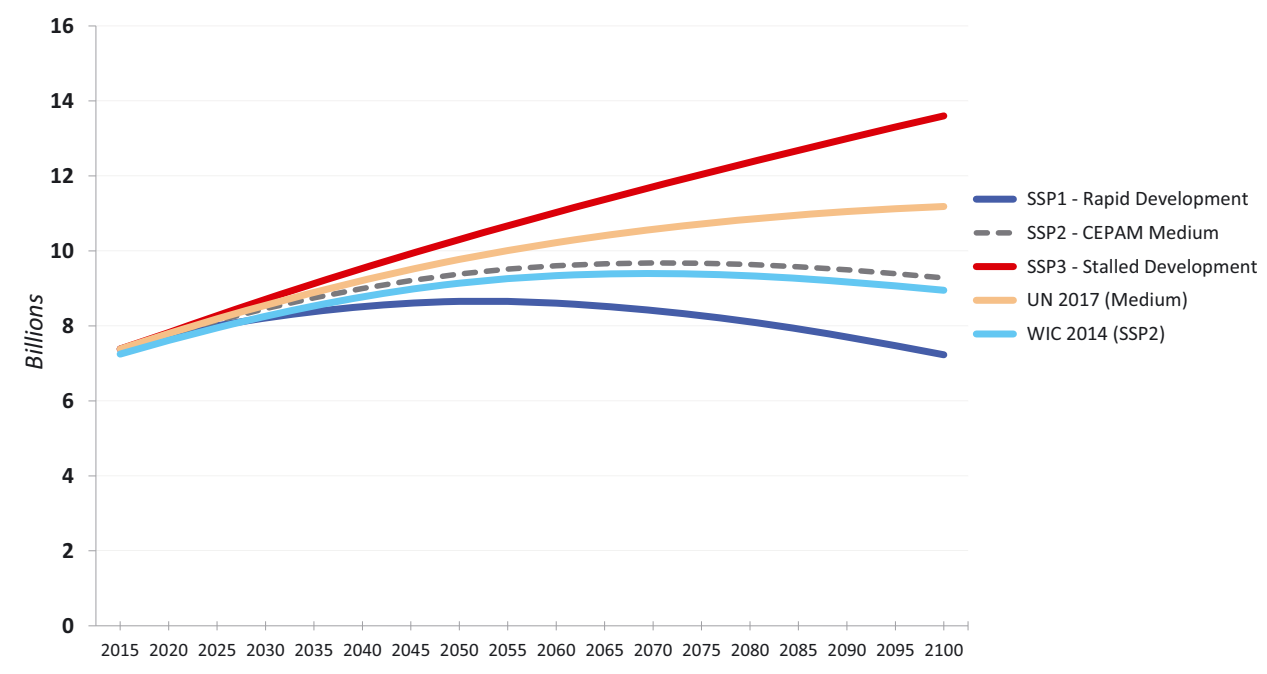

Source: Adapted from Lutz and KC (2018).

to $17 \%$ by mid-century, and to $29 \%$ by 2100 . Moreover, the faster gains in life expectancy assumed by the Rapid Social Development (SSP1) scenario result in the proportion of $65+$ reaching about $20 \%$ by mid-century, and $45 \%$ in 2100 .

\subsection{Regional trends: Sub-Saharan Africa}

CEPAM projections for world population have been revised upwards from the estimates in World population and human capital in the 21 st century (Lutz et al. 2014), primarily due to swifter declines in African mortality and slightly higher fertility rates than were previously assumed by other international assessments. In demographic terms, higher rates of child survival have the same effect as increasing fertility. Already by 2015 , the population was markedly higher than had been foreseen using the 2010 baseline data.

Spread of mass education has been very slow (with moderate progress in recent years) across Sub-Saharan Africa. Although educational attainment is expected to increase steadily over time in all of Africa's sub-regions, it will still remain lower than other continents at the aggregate level according to the CEPAM Medium (SSP2) scenario. While the average amount of schooling for western, middle, and eastern Africa stood between 5.4 and 5.8 years in 2015, these sub-regions are projected to reach between 9.3 and 9.9 years by 2060 . 
If development slows or stalls (SSP3) and female education does not expand, world population already crosses the 10 billion mark around 2045 and then continues to grow over the rest of the century to 13.6 billion in 2100. For Africa, such a path would likely be associated with weak resilience to environmental changes, high mortality, and widespread poverty. Interruptions in the spread of education would in fact accelerate population growth due to differing fertility behaviours between education groups.

In this stalled development scenario, the combination of high population growth with no further expansion of schooling results in African populations becoming less educated. Schooling expansion must keep pace with the pressures from rapid population growth, otherwise CEPAM results show hardly any improvement in the proportion of people without any formal education even at the global level - from $10 \%$ in 2015 to $9 \%$ by the end of the century.

\subsection{Regional trends: South Asia}

South Asia is the least educated region of Asia with the adult population averaging 6.6 years of schooling in 2015, or slightly above the average for Sub-Saharan Africa. Younger cohorts of Indian women have notably risen their levels of educational attainment. However, for the adult population of India as a whole, the low educational attainment of previous cohorts still overshadows the gains made in recent decades.

All of the projections foresee India eclipsing China to become the world's most populous country. And although most South Asian countries send large numbers of emigrants to North America, the EU, and Gulf countries, these flows have a negligible effect on population growth of the region. Still, by 2060 the median age in South Asia is expected to rise to a point similar to that of East Asia in 2015, stabilising by mid-century.

\subsection{Regional trends: East Asia}

The Japanese population has achieved global leadership in both educational attainment (ages 25+ averaged 13 years of schooling in 2015) and longevity (87.2 years for women in 2015-20, expected to climb to an impressive 95.7 by 2055-60). Japan is also further along in the ageing process, with the world's highest share of population 65 and older. The current proportion of people aged 65+ in Japan is 7\% higher than in the EU, and is expected to continue rising in the future as migration remains low and life expectancy increases.

China is rapidly catching up with the other highly educated East Asian countries after coming from far behind, and is predicted to reach 11.4 mean years of schooling in 2060. Over the same span of time, CEPAM results show China is expected to continue ageing to become 14\% smaller compared to itself in 2015. 


\subsection{Differing paths for international development}

Differences between CEPAM's projections make clear that changes in educational composition play a significant role in steering social development and population trends. The SSP3 pathway illustrates a future in which minimal investments in education and continued high fertility in Africa and parts of South Asia could reinforce each other, locking the world into a cycle of very high population growth, poverty, and high vulnerability.

In stark contrast, the SSP1 scenario shows how feedbacks between population, education, and development can alternatively result in further improvements in education and lower fertility. Many countries under this rapid development scenario would be well positioned to take advantage of the opportunities for broad economic and social advances that come from population structures shifting towards more workers and fewer dependents.

However, without sufficient education, the benefits of a maturing age structure are not guaranteed. To repeat the development successes seen in parts of East Asia and Latin America requires both prior investments in education and health infrastructure, along with positioning local enterprises to employ and retain the newfound potential of a more educated local labour force.

\section{Acknowledgements}

This summary of CEPAM's first major output is a modified version of Demographic and human capital scenarios for the 21st century: 2018 assessment for 201 countries: Executive Summary, a publication by IIASA and the Joint Research Centre (JRC), the European Commission's science and knowledge service.

Editors and authors of Demographic and human capital scenarios for the 21st century: 2018 assessment for 201 countries, the full volume: Wolfgang Lutz, Anne Goujon, Samir KC, Marcin Stonawski, and Nikolaos Stilianakis

Additional authors: Guy Abel, Bilal Barakat, Alain Bélanger, Alessandra Conte, Jakob Eder, Daniela Ghio, Erofili Grapsa, Sandra Jurasszovich, Elke Loichinger, Guillaume Marois, Michaela Potančoková, Patrick Sabourin, Tomáš Sobotka, Markus Speringer, Erich Striessnig, Marcus Wurzer, Dilek Yildiz, Sam Hyun Yoo and Kryštof Zeman

We thank Claudia Reiter for assistance with data and figures.

\section{References}

Loichinger, E. and G. Marois 2018. Education-specific labour force projections for EU-28 countries, Chapter 4. In: Demographic and human capital scenarios for the 21 st century: 2018 assessment for 201 countries, eds Lutz et al., 43-51. Luxembourg: Publications Office of the European Union. 
Lutz, W., W. P. Butz and S. KC (eds) 2014. World population and human capital in the twenty-first century. UK: Oxford University Press.

Lutz, W., A. Goujon, S. KC, M. Stonawski and N. Stilianakis (eds) 2018. Demographic and human capital scenarios for the 21st century: 2018 assessment for 201 countries. Luxembourg: Publications Office of the European Union.

Lutz, W. and S. KC 2018. Alternative scenarios for future world population growth, Chapter 11. In: Demographic and human capital scenarios for the 21st century: 2018 assessment for 201 countries, eds Lutz et al., 115-121. Luxembourg: Publications Office of the European Union.

Stonawski, M. 2018. Future population and human capital in the European union, Chapter 3. In: Demographic and human capital scenarios for the 21st century: 2018 assessment for 201 countries, eds Lutz et al., 29-41. Luxembourg: Publications Office of the European Union.

Appendix is on the following page. 


\section{Appendix: Population tables for select countries}

These snapshots of possible futures for India and Nigeria are taken as examples from a rich set of detailed projections. Interactive data accompanied by high-resolution colour graphs and population pyramids for all countries of the world can be found here: http://dataexplorer.wittgensteincentre.org/shiny/wic/

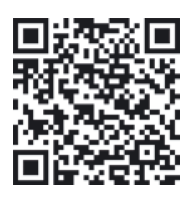

\section{India}

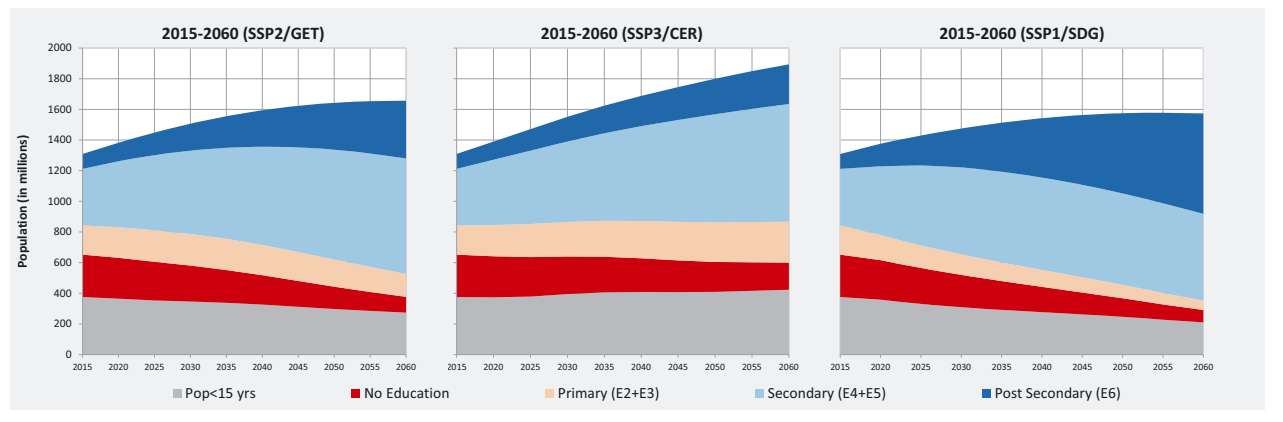

\section{Nigeria}
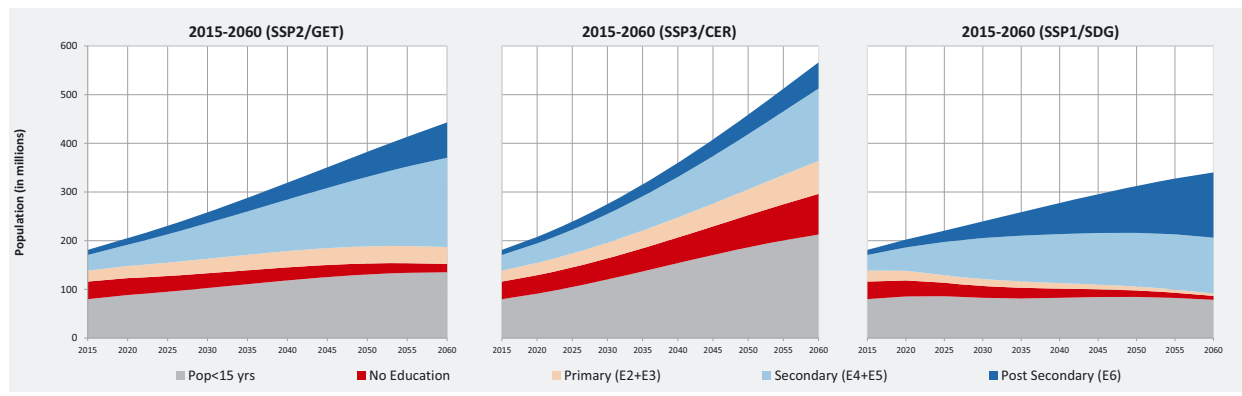